\title{
A HOEFFDING-TYPE INEQUALITY FOR ERGODIC TIME SERIES
}

\author{
YONGQIANG TANG \\ DEPARTMENT OF PSYCHIATRY, SUNY HEALTH SCIENCE CENTER
}

\begin{abstract}
In this paper, a Hoeffding-type inequality is presented for a class of ergodic time series. The inequality is then used to construct uniformly exponentially consistent tests, which are useful tools for studying Bayesian consistency.
\end{abstract}

\section{INTRODUCTION}

Hoeffding's (1963) inequality provides an exponential bound on the probability that the average of $n$ independent bounded random variables deviates from its mean. This inequality has been extended to martingales with bounded increments [Azuma (1967)] and functions with bounded differences [McDiarmid (1989)]; and see van de Geer (2002) for more discussions. These inequalities are of particular interest in applications in that the bounded probabilities are exponentially small for each finite $n$. Theorem 1 presents a simple version of the Azuma's inequality and includes Hoeffding's inequality as a special case when $X_{n}$ 's are independent.

Theorem 1. Let $\left\{X_{n}\right\}$ be a martingale difference sequence. Suppose that for each $i=1, \ldots, n, \alpha_{i} \leq X_{i} \leq \beta_{i}$ a.s., where $\alpha_{i}$ and $\beta_{i}$ are constants. Then, for all $n$ and $a>0$,

$$
\begin{aligned}
\operatorname{Pr}\left(\sum_{i=1}^{n} X_{n} \geq n a\right) & \leq \exp \left[\frac{-2 n^{2} a^{2}}{\sum_{i=1}^{n}\left(\beta_{i}-\alpha_{i}\right)^{2}}\right], \\
\operatorname{Pr}\left(\sum_{i=1}^{n} X_{n} \leq-n a\right) & \leq \exp \left[\frac{-2 n^{2} a^{2}}{\sum_{i=1}^{n}\left(\beta_{i}-\alpha_{i}\right)^{2}}\right] .
\end{aligned}
$$

In this paper, the Hoeffding's inequality is extended to a class of ergodic time series. The main idea for this generalization is to construct some bounded martingale difference sequence through the Poisson equation associated with a Markov process, which enables the use of Theorem 1, while the ergodic time series could be transformed into a Markov process using the technique of Herkenrath (2003).

The paper is organized as follows. Section 2 states the assumptions and establishes sufficient conditions under which the assumptions hold. The main inequality is presented in Section 3. Section 4 illustrates an application of the inequality to the construction of uniformly exponentially consistent tests which help to establish posterior consistency for nonlinear time series. Definition and properties of a uniformly ergodic Markov process are summarized in the Appendix.

Key words and phrases. Uniformly ergodic Markov processes, Poisson equation, Uniformly exponentially consistent tests. 


\section{Assumptions}

Consider a time-homogeneous stochastic process $\left\{X_{n}: n \geq-p+1\right\}$ with values in a measurable space $(\mathcal{X}, \mathcal{B})$ satisfying

$$
\operatorname{Pr}\left(X_{i+1} \in A \mid X_{t}, t \leq i\right)=\operatorname{Pr}\left(X_{i+1} \in A \mid X_{i-p+1}, \ldots, X_{i}\right),
$$

for all $A \in \mathcal{B}$ and $i \geq 0$, where $p \geq 1$ is a given integer. Let $Z_{i}=\left(X_{i-p+1}, \ldots, X_{i}\right)$ and $Y_{i}=Z_{p i}$. When $p=1, Y_{i}=Z_{i}=X_{i}$ for all $i$. Let $P\left(Z_{i} ; A\right)=\operatorname{Pr}\left(X_{i+1} \in\right.$ $\left.A \mid Z_{i}\right)$ and $P^{n}\left(Z_{0} ; A\right)=\operatorname{Pr}\left(X_{n} \in A \mid Z_{0}\right)$. Suppose that there exists a unique invariant probability measure $\pi$ for $\left\{X_{n}\right\}$. We make the following ergodicity assumption:

$$
\sum_{n=0}^{\infty} \sup _{Z_{0} \in \mathcal{X}^{p}}\left\|\frac{1}{p} \sum_{j=1}^{p} P^{n p+j}\left(Z_{0} ; \cdot\right)-\pi\right\|<\infty
$$

where $\|\cdot\|$ denotes the total variation norm. Let $R$ be a given value satisfying

$$
\sum_{n=0}^{\infty} \sup _{Z_{0}, Z_{0}^{*} \in \mathcal{X}^{p}}\left\|\frac{1}{p} \sum_{j=1}^{p} P^{n p+j}\left(Z_{0} ; \cdot\right)-\frac{1}{p} \sum_{j=1}^{p} P^{n p+j}\left(Z_{0}^{*} ; \cdot\right)\right\| \leq R .
$$

Assumption (1) and the triangle inequality imply $R<\infty$.

When $p=1,\left\{X_{n}\right\}$ is a Markov process. If (1) holds, clearly

$$
\lim _{n \rightarrow \infty} \sup _{X_{0} \in \mathcal{X}}\left\|P^{n}\left(X_{0} ; \cdot\right)-\pi\right\|=0,
$$

that is, $\left\{X_{n}\right\}$ is uniformly ergodic. The converse holds by Lemma 4 , which also helps to calculate $R$.

When $p \geq 2$, the stochastic process is generally not Markovian. However, both the multivariate series $\left\{Z_{n}: n \geq 0\right\}$ and $\left\{Y_{n}: n \geq 0\right\}$ are Markov processes. We study $\left\{Y_{n}\right\}$ because $\left\{Z_{n}\right\}$ may not be suitable to study the ergodicity of $\left\{X_{n}\right\}$ when $\left\{Y_{n}\right\}$ is uniformly ergodic, as noted by Herkenrath (2003). Denote the transition probability, $n$-step transition probability, and the invariant probability measure of $\left\{Y_{n}\right\}$ by $Q\left(Y_{i} ; B\right)=\operatorname{Pr}\left(Y_{i+1} \in B \mid Y_{i}\right), Q^{n}\left(Y_{0} ; B\right)=\operatorname{Pr}\left(Y_{n} \in B \mid Y_{0}\right)$ and $\pi_{Q}$ respectively, where $B \in \mathcal{B}^{p}$. Obviously

$$
Q\left(Y_{i} ; B\right)=\int_{B} P\left(Z_{i p} ; d X_{i p+1}\right) \ldots P\left(Z_{i p+p-1} ; d X_{i p+p}\right) .
$$

Lemma 1 says that (1) holds if $\left\{Y_{n}\right\}$ is uniformly ergodic. Lemma 2 mimics Lemma 3 of Herkenrath (2003) and provides an upper bound on $R$ under a condition which may be easily verified.

Lemma 1. If the Markov process $\left\{Y_{n}\right\}$ is uniformly ergodic, then the left-hand side of (1) is bounded by $\sum_{n=1}^{\infty} \sup _{Y_{0} \in \mathcal{X}^{p}}\left\|Q^{n}\left(Y_{0} ; \cdot\right)-\pi_{Q}\right\|<\infty$, and an upper bound of $R$ in (2) is given by $\sum_{n=1}^{\infty} \sup _{Y_{0}, Y_{0}^{*} \in \mathcal{X}^{p}}\left\|Q^{n}\left(Y_{0} ; \cdot\right)-Q^{n}\left(Y_{0}^{*} ; \cdot\right)\right\|<\infty$.

Proof. Herkenrath (2003) showed that the marginal measures of $\pi_{Q}$ are identical to $\pi$ when $\left\{Y_{n}\right\}$ is uniformly ergodic. For any function $g$, define $G\left(Y_{n}\right)=$ $\sum_{j=1}^{p} g\left(X_{n p-p+j}\right), \pi(g)=\int g(X) \pi(d X)$ and $\pi_{Q}(G)=\int G(Y) \pi_{Q}(d Y)=p \pi(g)$. 
Then, for each $n \geq 0$,

$$
\begin{aligned}
\sup _{Z_{0} \in \mathcal{X}^{p}}\left\|\frac{1}{p} \sum_{j=1}^{p} P^{n p+j}\left(Z_{0} ; \cdot\right)-\pi\right\| & =\sup _{\substack{g:|g| \leq 1 \\
Z_{0} \in \mathcal{X}^{p}}}\left|\frac{1}{p} \sum_{j=1}^{p} \mathrm{E}\left(g\left(X_{n p+j}\right) \mid Z_{0}\right)-\pi(g)\right| \\
& =\sup _{\substack{g:|g| \leq 1 \\
Z_{0} \in \mathcal{X}^{p}}}\left|\frac{1}{p} \mathrm{E}\left(G\left(Y_{n+1}\right) \mid Z_{0}\right)-\frac{1}{p} \pi_{Q}(G)\right| \\
& \leq \sup _{Y_{0}=Z_{0} \in \mathcal{X}^{p}}\left\|Q^{n+1}\left(Y_{0} ; \cdot\right)-\pi_{Q}\right\| .
\end{aligned}
$$

The bound on the left-hand side of (1) is obtained by summing over $n \geq 0$. The upper bound on $R$ could be proved similarly. Moreover, the bounds are finite when $\left\{Y_{n}\right\}$ is uniformly ergodic by Lemma 4 .

Lemma 2. If there exists a $\delta>0$ and a probability measure $\mu$ on $(\mathcal{X}, \mathcal{B})$ such that for all $A \in \mathcal{B}$,

$$
\inf _{Z_{0} \in \mathcal{X}^{p}} P\left(Z_{0} ; A\right) \geq \delta \mu(A) .
$$

then the Markov process $\left\{Y_{n}\right\}$ is uniformly ergodic, and $R \leq \delta^{-p}-1$ in (2).

Proof. Let $\nu(d Y)=\prod_{j=1}^{p} \mu\left(d X_{j}\right)$ be the product measure. For all $B$ of the form $B=A_{1} \times \ldots \times A_{p}$, it is easy to show

$$
Q\left(Y_{i} ; B\right)=\int_{B} P\left(Z_{i p} ; d X_{i p+1}\right) \ldots P\left(Z_{i p+p-1} ; d X_{i p+p}\right) \geq \delta^{p} \prod_{j=1}^{p} \mu\left(A_{j}\right)=\delta^{p} \nu(B) .
$$

This implies that $Q\left(Y_{i} ; B\right) \geq \delta^{p} \nu(B)$ for any $B \in \mathcal{B}^{p}$. Hence $\left\{Y_{n}\right\}$ is uniformly ergodic by Lemma 1. By Lemma 4 and Lemma 1 ,

$$
R \leq \sum_{i=1}^{\infty} \sup _{Y_{0}, Y_{0}^{*} \in \mathcal{X}^{p}}\left\|Q^{n}\left(Y_{0} ; \cdot\right)-Q^{n}\left(Y_{0}^{*} ; \cdot\right)\right\| \leq \delta^{-p}-1 .
$$

\section{MAIN INEQUALITY}

For a $\pi$ integrable function $g, G\left(Y_{i}\right)=\sum_{j=1}^{p} g\left(X_{i p-p+j}\right)$ and $\pi(g)=\int g(X) \pi(d X)$, consider the Poisson equation

$$
\breve{G}(Y)-\mathrm{E}\left(\breve{G}\left(Y_{1}\right) \mid Y_{0}=Y\right)=G(Y)-p \pi(g) .
$$

If $\breve{G}$ solves (4), then the partial sum

$$
S_{m p}(\bar{g})=\sum_{i=1}^{m p}\left[g\left(X_{i}\right)-\pi(g)\right]=\sum_{i=1}^{m}\left[G\left(Y_{i}\right)-p \pi(g)\right]
$$

could be written as

$$
S_{m p}(\bar{g})=\sum_{i=1}^{m}\left[\breve{G}\left(Y_{i}\right)-\mathrm{E}\left(\breve{G}\left(Y_{i+1}\right) \mid Y_{i}\right)\right]=M_{m}(\breve{G})+R_{m}(\breve{G})
$$

for all $m \geq 1$, where $M_{m}(\breve{G})=\sum_{i=1}^{m}\left[\breve{G}\left(Y_{i}\right)-\mathrm{E}\left(\breve{G}\left(Y_{i}\right) \mid Y_{i-1}\right)\right]$ is a martingale, and $R_{m}(\breve{G})=\mathrm{E}\left(\breve{G}\left(Y_{1}\right) \mid Y_{0}\right)-\mathrm{E}\left(\breve{G}\left(Y_{1}\right) \mid Y_{0}=Y_{m}\right)$. See Meyn and Tweedie (1993) for details on the technique of constructing martingale via the solution to Poisson 
equation. The follow lemma says that (4) admits a uniformly bounded solution if $g$ is a bounded function under the assumption (1). Thus $R_{m}(\breve{G})$ and each term in $M_{m}(\breve{G})$ are uniformly bounded, which enables the use of Theorem 1.

Lemma 3. If (1) and (2) hold, and $l \leq g(x) \leq u$ for any $x \in \mathcal{X}$, then

$$
\breve{G}(Y)=\sum_{i=0}^{\infty}\left[\mathrm{E}\left(G\left(Y_{i}\right) \mid Y_{0}=Y\right)-p \pi(g)\right]=\sum_{i=-p+1}^{\infty}\left[\mathrm{E}\left(g\left(X_{i}\right) \mid Y_{0}=Y\right)-\pi(g)\right]
$$

is a uniformly bounded solution of (4) and satisfies

$$
\sup _{Z_{0}, Z_{0}^{*} \in \mathcal{X}^{p}}\left|\mathrm{E}\left(\breve{G}\left(Y_{1}\right) \mid Y_{0}=Z_{0}\right)-\mathrm{E}\left(\breve{G}\left(Y_{1}\right) \mid Y_{0}=Z_{0}^{*}\right)\right| \leq p(u-l) R / 2 .
$$

Proof. Let $g^{*}(x)=g(x)-(u+l) / 2$. Then $\left|g^{*}(x)\right| \leq(u-l) / 2$ for any $x$ and

$$
\begin{aligned}
|\breve{G}(Y)| & \leq\left|\sum_{i=-p+1}^{0} g\left(X_{i}\right)-p \pi(g)\right|+\left|\sum_{i=1}^{\infty}\left[\mathrm{E}\left(g\left(X_{i}\right) \mid Y_{0}=Y\right)-\pi(g)\right]\right| \\
& \leq \sum_{i=-p+1}^{0}\left|g^{*}\left(X_{i}\right)-\pi\left(g^{*}\right)\right|+\left|\sum_{i=1}^{\infty}\left[\mathrm{E}\left(g^{*}\left(X_{i}\right) \mid Y_{0}=Y\right)-p \pi\left(g^{*}\right)\right]\right| \\
& \leq p(u-l)+\frac{p(u-l)}{2} \sum_{n=0}^{\infty} \sup _{Z_{0} \in \mathcal{X}^{p}}\left\|\frac{1}{p} \sum_{j=1}^{p} P^{n p+j}\left(Z_{0} ; \cdot\right)-\pi\right\| .
\end{aligned}
$$

Hence $\breve{G}(Y)$ is uniformly bounded and is well-defined.

Note that

$$
\breve{G}(Y)=\sum_{i=0}^{\infty}\left[\mathrm{E}\left(G\left(Y_{i}\right) \mid Y_{0}=Y\right)-p \pi(g)\right]=\sum_{i=1}^{\infty}\left[\mathrm{E}\left(G\left(Y_{i}\right) \mid Y_{1}=Y\right)-p \pi(g)\right],
$$

and hence

$$
\mathrm{E}\left(\breve{G}\left(Y_{1}\right) \mid Y_{0}=Y\right)=\sum_{i=1}^{\infty}\left[\mathrm{E}\left(G\left(Y_{i}\right) \mid Y_{0}=Y\right)-p \pi(g)\right] .
$$

Thus $\breve{G}(Y)$ is a solution of (4) since

$$
\begin{aligned}
& \breve{G}(Y)-\mathrm{E}\left(\breve{G}\left(Y_{1}\right) \mid Y_{0}=Y\right) \\
= & \sum_{i=0}^{\infty}\left[\mathrm{E}\left(G\left(Y_{i}\right) \mid Y_{0}=Y\right)-p \pi(g)\right]-\sum_{i=1}^{\infty}\left[\mathrm{E}\left(G\left(Y_{i}\right) \mid Y_{0}=Y\right)-p \pi(g)\right] \\
= & G(Y)-p \pi(g) .
\end{aligned}
$$


Now for any $Z_{0}$ and $Z_{0}^{*}$,

$$
\begin{aligned}
& \left|\mathrm{E}\left(\breve{G}\left(Y_{1}\right) \mid Y_{0}=Z_{0}\right)-\mathrm{E}\left(\breve{G}\left(Y_{1}\right) \mid Y_{0}=Z_{0}^{*}\right)\right| \\
= & \left|\sum_{i=1}^{\infty}\left[\mathrm{E}\left(G\left(Y_{i}\right) \mid Y_{0}=Z_{0}\right)-\mathrm{E}\left(G\left(Y_{i}\right) \mid Y_{0}=Z_{0}^{*}\right)\right]\right| \\
= & \left|\sum_{i=1}^{\infty}\left[\mathrm{E}\left(g^{*}\left(X_{i}\right) \mid Y_{0}=Z_{0}\right)-\mathrm{E}\left(g^{*}\left(X_{i}\right) \mid Y_{0}=Z_{0}^{*}\right)\right]\right| \\
\leq & \frac{p(u-l)}{2} \sum_{n=0}^{\infty}\left\|\frac{1}{p} \sum_{j=1}^{p} P^{n p+j}\left(Z_{0} ; \cdot\right)-\frac{1}{p} \sum_{j=1}^{p} P^{n p+j}\left(Z_{0}^{*} ; \cdot\right)\right\| \\
\leq & p(u-l) R / 2 .
\end{aligned}
$$

We are now in a position to present our main result.

Theorem 2. If (1) and (2) hold, and $l \leq g(x) \leq u$ for any $x \in \mathcal{X}$, then

$$
\begin{aligned}
& \operatorname{Pr}\left(\sum_{i=1}^{n}\left[g\left(X_{i}\right)-\pi(g)\right] \geq n a\right) \leq \exp \left[\frac{-2(n a-(u-l)(R p / 2+k))^{2}}{m(R+1)^{2} p^{2}(u-l)^{2}}\right], \\
& \operatorname{Pr}\left(\sum_{i=1}^{n}\left[g\left(X_{i}\right)-\pi(g)\right] \leq-n a\right) \leq \exp \left[\frac{-2(n a-(u-l)(R p / 2+k))^{2}}{m(R+1)^{2} p^{2}(u-l)^{2}}\right],
\end{aligned}
$$

for all $a>0$ and $n \geq(R p / 2+k)(u-l) / a$, where $m=\left[\frac{n}{p}\right], k=n-m p$ satisfying $0 \leq k<p$. Furthermore, the following hold

$$
\begin{aligned}
& \operatorname{Pr}\left(\sum_{i=1-p}^{n}\left[g\left(X_{i}\right)-\pi(g)\right] \geq(n+p) a\right) \leq \exp \left[\frac{-2\left(n a+p a-R^{*}\right)^{2}}{(m+1)(R+1)^{2} p^{2} b^{2}}\right], \\
& \operatorname{Pr}\left(\sum_{i=1-p}^{n}\left[g\left(X_{i}\right)-\pi(g)\right] \leq-(n+p) a\right) \leq \exp \left[\frac{-2\left(n a+p a-R^{*}\right)^{2}}{(m+1)(R+1)^{2} p^{2} b^{2}}\right],
\end{aligned}
$$

for all $a>0$ and $n \geq R^{*} / a-p$, where $b=u-l$ and $R^{*}=(R p / 2+p) b$.

Proof. Let $S_{n}(\bar{g})=\sum_{i=1}^{n}\left[g\left(X_{i}\right)-\pi(g)\right]$. Then $S_{n}(\bar{g})$ could be written as

$$
S_{n}(\bar{g})=M_{m}(\breve{G})+R_{m}(\breve{G})+\sum_{j=1}^{k}\left(g\left(X_{m p+j}\right)-\pi(g)\right),
$$

where $M_{m}(\breve{G})$ and $R_{m}(\breve{G})$ are defined in (5). Note that $R_{m}(\breve{G})$ is bounded by $R p(u-l) / 2$ by Lemma $3,\left|\sum_{j=1}^{k}\left(g\left(X_{m p+j}\right)-\pi(g)\right)\right|$ is bounded by $k(u-l)$. Thus

$$
\operatorname{Pr}\left(\sum_{i=1}^{n}\left[g\left(X_{i}\right)-\pi(g)\right] \geq n a\right) \leq \operatorname{Pr}\left(M_{m}(\breve{G}) \geq n a-(R p / 2+k)(u-l)\right),
$$

and

$$
\operatorname{Pr}\left(\sum_{i=1}^{n}\left[g\left(X_{i}\right)-\pi(g)\right] \leq-n a\right) \leq \operatorname{Pr}\left(M_{m}(\breve{G}) \leq(R p / 2+k)(u-l)-n a\right) .
$$


Note that for each $i=1, \ldots, m$,

$\breve{G}\left(Y_{i}\right)-\mathrm{E}\left(\breve{G}\left(Y_{i}\right) \mid Y_{i-1}\right)=G\left(Y_{i}\right)-p \pi(g)+\mathrm{E}\left(\breve{G}\left(Y_{1}\right) \mid Y_{0}=Y_{i}\right)-\mathrm{E}\left(\breve{G}\left(Y_{i}\right) \mid Y_{i-1}\right)$.

Thus by Lemma 3 ,

$$
-\frac{(R p+p)(u-l)}{2}-p \pi(g) \leq \breve{G}\left(Y_{i}\right)-\mathrm{E}\left(\breve{G}\left(Y_{i}\right) \mid Y_{i-1}\right) \leq \frac{(R p+p)(u-l)}{2}-p \pi(g) \text {. }
$$

Since $M_{m}(\breve{G})$ is the sum of bounded martingale difference sequence, an application of Theorem 1 yields (6) and (7).

To show (8) and (9), we note that

$$
S_{n}^{*}(\bar{g})=\sum_{i=-p+1}^{n}\left[g\left(X_{i}\right)-\pi(g)\right]=M_{m}^{*}(\breve{G})+R_{m}^{*}(\breve{G})+\sum_{j=1}^{k}\left(g\left(X_{m p+j}\right)-\pi(g)\right),
$$

where $R_{m}^{*}(\breve{G})=\breve{G}\left(Y_{0}\right)-\breve{G}\left(Y_{m+1}\right)$ and $M_{m}^{*}(\breve{G})=\sum_{i=1}^{m+1}\left[\breve{G}\left(Y_{i}\right)-E\left(\breve{G}\left(Y_{i}\right) \mid Y_{i-1}\right)\right]$.

Note that

$$
\begin{aligned}
& \left|R_{m}^{*}(\breve{G})+\sum_{j=1}^{k}\left(g\left(X_{m p+j}\right)-\pi(g)\right)\right| \\
= & \left|\sum_{i=1-p}^{0} g\left(X_{i}\right)-\sum_{i=m p+k+1}^{m p+p} g\left(X_{i}\right)-k \pi(g)+\mathrm{E}\left(\breve{G}\left(Y_{1}\right) \mid Y_{0}\right)-\mathrm{E}\left(\breve{G}\left(Y_{1}\right) \mid Y_{0}=Y_{m+1}\right)\right|
\end{aligned}
$$

is bounded by $R p(u-l) / 2+p(u-l)$. Similarly, (8) and (9) hold by Theorem 1 .

Remark 1. All inequalities in Theorem 2 do not depend on the initial distribution of $Y_{0}$. The inequalities (6) and (7) are preferred when $Y_{0}$ is assumed to be fixed while (8) and (9) are preferred when $Y_{0}$ is assumed to be random.

Corollary 3. Suppose that $\left\{X_{n}: n \geq 0\right\}$ is a uniformly ergodic Markov process, that is, there exists $\delta>0$ and a probability measure $\nu$ such that for all $A \in \mathcal{B}$,

$$
\inf _{x \in \chi} P^{m}(x, A) \geq \delta \nu(A) .
$$

Let $g: \mathcal{X} \rightarrow[l, u]$ be a measurable function. Then

$$
\begin{aligned}
\operatorname{Pr}\left(\sum_{i=1}^{n}\left[g\left(X_{i}\right)-\pi(g)\right] \geq n a\right) & \leq \exp \left[\frac{-2(n a-R(u-l) / 2)^{2}}{n(R+1)^{2}(u-l)^{2}}\right], \\
\operatorname{Pr}\left(\sum_{i=1}^{n}\left[g\left(X_{i}\right)-\pi(g)\right] \leq-n a\right) & \leq \exp \left[\frac{-2(n a-R(u-l) / 2)^{2}}{n(R+1)^{2}(u-l)^{2}}\right],
\end{aligned}
$$

for all $a>0$ and $n \geq R(u-l) /(2 a)$, where $R=\rho /(1-\rho)$ and $\rho=(1-\delta)^{1 / m}$. Furthermore, the following hold

$$
\begin{aligned}
\operatorname{Pr}\left(\sum_{i=0}^{n}\left[g\left(X_{i}\right)-\pi(g)\right] \geq(n+1) a\right) & \leq \exp \left[\frac{-2\left(n a+a-R^{*}\right)^{2}}{(n+1)(R+1)^{2}(u-l)^{2}}\right], \\
\operatorname{Pr}\left(\sum_{i=0}^{n}\left[g\left(X_{i}\right)-\pi(g)\right] \leq-(n+1) a\right) & \leq \exp \left[\frac{-2\left(n a+a-R^{*}\right)^{2}}{(n+1)(R+1)^{2}(u-l)^{2}}\right],
\end{aligned}
$$

for all $a>0$ and $n \geq R^{*} / a-1$, where $R^{*}=(R / 2+1)(u-l)$. 


\section{Applichtion}

In this section, we use the Hoeffding-type inequality to construct uniformly exponentially consistent tests, which are useful tools for studying Bayesian consistency; see Schwartz (1965).

Let $X^{n}=\left\{X_{-p+1}, \ldots, X_{n}\right\}$ denote the observations from the $p$-th order autoregressive model with transition density $f_{0}\left(X_{i} \mid Z_{i-1}\right)$, where $Z_{i}=\left(X_{i-p+1}, \ldots, X_{i}\right)$. Let $\Pi$ be a prior on the transition density $f$ in a Bayesian nonparametric procedure. Assume that there is a unique invariant distribution $\pi_{p f}$ of $Z_{i}$ associated with each $f$ in the support of the prior. Denote the marginal distribution of $\pi_{p f}$ by $\pi_{1 f}$.

Example 1. Consider the following nonparametric mixture model,

$$
f_{P}\left(X_{i} \mid Z_{i-1}\right)=\int \frac{1}{\sqrt{2 \pi}} \exp \left(-\frac{\left(X_{i}-H\left(\boldsymbol{\theta}, Z_{i-1}\right)\right)^{2}}{2}\right) d P(\boldsymbol{\theta}),
$$

where $H$ is a continuous function bounded by $a$ and $P$ is an unknown distribution function. A prior on the transition density is induced from that on $P$. For any $P$,

$$
f_{P}\left(X_{i} \mid Z_{i-1}\right) \geq q\left(X_{i}\right)=\frac{1}{\sqrt{2 \pi}} \exp \left(-X_{i}^{2}-a^{2}\right)=c q\left(X_{i}\right) / c
$$

where $c=\int q\left(X_{i}\right) d X_{i}=\exp \left(-a^{2}\right) / \sqrt{2}<1$, and $q\left(X_{i}\right) / c$ is a probability density function. By Lemma $2, R$ in (2) is uniformly bounded by $c^{-p}-1$ for any $P$.

Let $f_{0}$ be a specific transition density. Consider testing $H_{0}: f=f_{0}$ versus $f \in V^{c}$ where $V=\left\{f: \pi_{1 f}(g)<\pi_{1 f_{0}}(g)+\epsilon\right\}, \epsilon>0$ and $g$ is a bounded continuous function. Without loss of generality, we assume $0 \leq g \leq 1$. We shall construct a sequence of uniformly exponentially consistent tests for the above testing problem. If $R$ in (2) is uniformly bounded by $R_{u}<\infty$ for any $f \in \Pi$, then

$$
\phi_{n}\left(X^{n}\right)=I\left(n^{-1} \sum_{i=1}^{n} g_{1}\left(X_{i}\right)>\pi_{1 f_{0}}(g)+\epsilon / 2\right)
$$

where $I$ is the indicator function, is such a sequence of exponentially consistent tests since by theorem 2 , when $n \geq 4\left(R_{u} / 2+1\right) p / \epsilon$,

$$
\mathrm{E}_{f_{0}}\left(\phi_{n}\right)=\operatorname{Pr}_{f_{0}}\left[\sum_{i=1}^{n}\left(g\left(X_{i}\right)-\pi_{1 f_{0}}(g)\right)>\frac{n \epsilon}{2}\right] \leq \exp (-n \beta)
$$

and

$$
\sup _{f \in V^{c}} \mathrm{E}_{f}\left(1-\phi_{n}\right) \leq \sup _{f \in V^{c}} \operatorname{Pr}_{f}\left[\sum_{i=1}^{n}\left(g\left(X_{i}\right)-\pi_{1 f}(g)\right)<-\frac{n \epsilon}{2}\right] \leq \exp (-n \beta),
$$

where $\beta=\epsilon^{2} /\left(8 R_{u} p^{2}+8 p^{2}\right)$. Note that $V$ forms a subbase of the weak topology at $f_{0}$. Hence uniformly exponentially consistent tests for $H_{0}: f=f_{0}$ versus $f \in U^{c}$, where $U$ is a weak neighborhood of $f_{0}$, may be easily constructed from the above tests.

\section{APPENDIX}

Definition 1. A Markov process $\left\{\phi_{n}: n \geq 0\right\}$ with values in a measurable space $(W, \mathcal{W})$ is called uniformly ergodic if $\lim _{n \rightarrow \infty} \sup _{\phi_{0} \in W}\left\|P^{n}\left(\phi_{0} ; \cdot\right)-\pi\right\|=0$, where $P^{n}\left(\phi_{0} ; \cdot\right)$ is its $n$-step transition probability measure and $\pi$ is the invariant probability measure. 
Lemma 4. The process $\left\{\phi_{n}\right\}$ is uniformly ergodic if and only if there exists a positive integer $m, \delta>0$ and a probability measure $\nu$ such that $\inf _{\phi_{0} \in W} P^{m}\left(\phi_{0}, A\right) \geq$ $\delta \nu(A)$, for all $A \in \mathcal{W}$. Moreover, the following inequalities hold,

$$
\begin{aligned}
\sup _{\phi_{0} \in W}\left\|P^{n}\left(\phi_{0}, \cdot\right)-\pi\right\| & \leq \rho^{n}, \\
\sup _{\phi_{0}, \phi_{0}^{*} \in W}\left\|P^{n}\left(\phi_{0}, \cdot\right)-P^{n}\left(\phi_{0}^{*}, \cdot\right)\right\| & \leq \rho^{n}, \\
\sum_{n=1}^{\infty} \sup _{\phi_{0} \in W}\left\|P^{n}\left(\phi_{0}, \cdot\right)-\pi\right\| & \leq \rho /(1-\rho), \\
\sum_{n=1}^{\infty} \sup _{\phi_{0}, \phi_{0}^{*} \in W}\left\|P^{n}\left(\phi_{0}, \cdot\right)-P^{n}\left(\phi_{0}^{*}, \cdot\right)\right\| & \leq \rho /(1-\rho),
\end{aligned}
$$

where $\rho=(1-\delta)^{1 / m}$.

Proof. The first part of the lemma is a re-statement of Theorem 16.0.2 of Meyn and Tweedie (1993). Meyn and Tweedie (1993) used the coupling method to prove (10) in their Theorem 16.2.4. Their proof in fact leads to

$$
\left\|\int P^{n}\left(\phi_{0} ; \cdot\right) d \lambda\left(\phi_{0}\right)-\int P^{n}\left(\phi_{0} ; \cdot\right) d \mu\left(\phi_{0}\right)\right\| \leq(1-\delta)^{n / m},
$$

where $\lambda$ and $\mu$ are two different initial distributions of $\phi_{0}$. If $\lambda$ is degenerate at $\phi_{0}$ and $\mu$ is $\pi$, (12) reduces to (10). If $\lambda$ is degenerate at $\phi_{0}$ and $\mu$ is degenerate at $\phi_{0}^{*}$, (12) reduces to (11). Inequalities (12) and (13) hold given (10) and (11).

Acknowledgments. The work is based on part of the author's Ph.D. dissertation submitted to North Carolina State University. The author is deeply indebted to Professor Subhashis Ghosal for his guidance and invaluable discussions during the course of the work and for his suggestions for improvement.

\section{REFERENCES}

Azuma, K. (1967). Weighted sums of certain dependent random variables. Tôhoku Mathematical Journal 19(3) 357-367.

Herkenrath, U. (2003). On the uniform ergodicity of Markov processes of order 2. Journal of Applied Probability 40(2) 455-472.

McDiarmid, C. (1989). On the method of bounded differences. In Surveys in Combinatorics (J. Principe, L. Gile, N. Morgan and E. Wilson, eds.). Cambridge University Press, Cambridge.

Meyn, S. P. and Tweedie, R. L. (1993). Markov Chains and Stochastic Stability. Springer, New York.

Schwartz, L. (1965). On Bayes procedures. Z. Wahr. Verw. Gebiete 4 10-26.

VAN DE GEeR, S. (2002). On Hoeffding's inequality for dependent random variables. In Empirical Process Techniques for Dependent Data (H. Dehling, T. Mikosch and M. Srensen, eds.). Birkhuser, Boston.

Department of Psychiatry, SUny Health Science Center, 450 Clarkson Avenue, Box 1203, BRookLYN, NY 11203, USA

E-mail address: yongqiang_tang@yahoo.com 